\title{
Special section on stochastic and quantum computing
}

The need for efficient computing is growing rapidly in conjunction with the expansion of application areas of computer technology. As indicated by recent studies, efficient computing thanks to stochasticism is indispensable for real-world applications. Also, based on the remarkable progress of quantum devices, real parallel processing utilizing quantum mechanical properties is a promising approach to achieving ultimate efficiency.

This special section is organized to transmit the latest results related to stochastic and quantum computing. It consists of two invited papers and four peer-reviewed, regular papers from various fields, including quantum annealing, stochastic computing, quantumdot cellular automata, and neural networks. This section is sure to provide those who study these topics with new ideas and findings.

On behalf of the editorial committee, I would like to express my sincere thanks to all authors for their contributions and to the anonymous reviewers for their hard work within a tight schedule. I would also like to thank the editorial committee members and editorial staff of NOLTA journal for their continuous support and efforts. I hope that this special section contributes to further research progress in related fields.

\section{Shigeo Sato \\ Guest Editor}

\section{Editorial Committee of the Special Section}

\section{Guest Editor:}

Shigeo Sato (Tohoku University)

\author{
Associate Editors: \\ Tetsuya Asai (Hokkaido University) \\ Igor Belykh (Georgia State University) \\ Jozef Gruska (Masaryk University) \\ Yoshinori Hayakawa (Tohoku University) \\ Mitsunaga Kinjo (University of the Ryukyus) \\ Jordi Madrenas (Technical University of Catalunya) \\ Masaaki Maezawa (National Institute of Advanced Industrial Science and Technology) \\ Koji Nakajima (Tohoku University) \\ Tohru Nitta ((National Institute of Advanced Industrial Science and Technology) \\ Takeshi Onomi (Fukuoka Institute of Technology)
}

\section{Secretary of the Special Section:}

Shigeo Sato (Tohoku University)

\section{Advisory:}

Yoshihiko Horio (Tohoku University) 